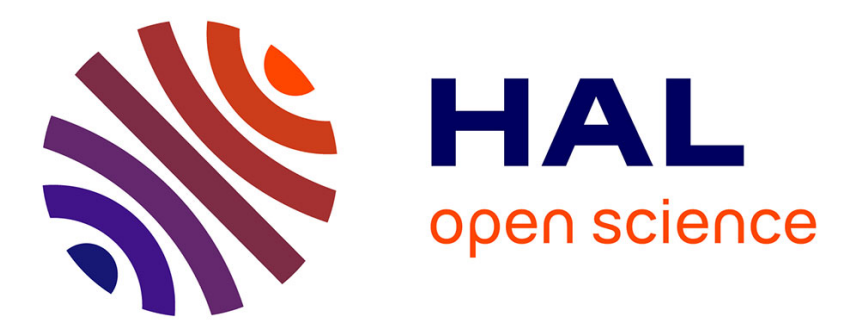

\title{
Comparison of two methods for unsupervised person identification in TV shows
}

Paul Gay, Grégor Dupuy, Carole Lailler, Jean-Marc Odobez, Sylvain Meignier, Paul Deléglise

\section{- To cite this version:}

Paul Gay, Grégor Dupuy, Carole Lailler, Jean-Marc Odobez, Sylvain Meignier, et al.. Comparison of two methods for unsupervised person identification in TV shows. 12th International Workshop on Content-Based Multimedia Indexing, 2014, Klagenfurt, Austria. pp.1 - 6, 10.1109/CBMI.2014.6849828 . hal-01433260

\section{HAL Id: hal-01433260 \\ https://hal.science/hal-01433260}

Submitted on 1 Apr 2017

HAL is a multi-disciplinary open access archive for the deposit and dissemination of scientific research documents, whether they are published or not. The documents may come from teaching and research institutions in France or abroad, or from public or private research centers.
L'archive ouverte pluridisciplinaire $\mathbf{H A L}$, est destinée au dépôt et à la diffusion de documents scientifiques de niveau recherche, publiés ou non, émanant des établissements d'enseignement et de recherche français ou étrangers, des laboratoires publics ou privés. 


\title{
Comparison of Two Methods for Unsupervised Person Identification in TV Shows
}

\author{
Paul Gay*†, Grégor Dupuy*, Carole Lailler*, Jean-Marc Odobez ${ }^{\dagger}$, Sylvain Meignier*, and Paul Deléglise** ${ }^{*}$ LUNAM \\ Université, LIUM, Le Mans, France \\ †IDIAP Research Institute, Martigny, Switzerland
}

\begin{abstract}
We address the task of identifying people appearing in TV shows. The target persons are all people whose identity is said or written, like the journalists and the well known people, as politicians, athletes, celebrities, etc. In our approach, overlaid names displayed on the images are used to identify the persons without any use of biometric models for the speakers and the faces. Two identification methods are evaluated as part of the REPERE French evaluation campaign. The first one relies on co-occurrence times between overlay person names and speaker/face clusters, and rule-based decisions which assign a name to each monomodal cluster. The second method uses a Conditionnal Random Field (CRF) which combine different types of co-occurrence statistics and pair-wised constraints to jointly identify speakers and faces.
\end{abstract}

\section{INTRODUCTION}

The main purpose of this paper is to answer the questions "who is speaking?" and "who is seen?" at any time of the videos. The target persons are both journalists and guests, which can refer either to experts in a specific field, or to politicians, or celebrities. In other words, we try to identify by their names the people of a video using only the information of the video, without any biometric model related to the target persons.

Without any a priori information, person identities can be retrieved either from the speech transcripts or from the overlaid person names (OPN) commonly used to introduce the current speaker (e.g. see Fig 1). Several speaker identification ap-

Fig. 1. Example of annotation with an OPN introducing the speaker.

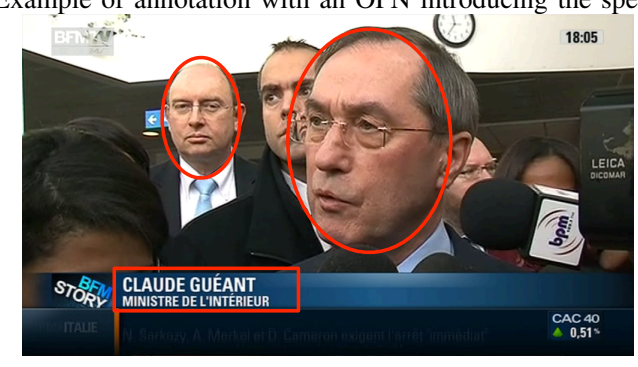

Head: Claude Géuant, unknown

OPN: Claude Géant

Speaker: Claude Géant

proaches using speech transcripts have already been presented in [1][2][3]. Person identification from transcripts generally gives good results when transcripts are well segmented and close to what was said. However, results deteriorates as soon as automatic transcripts, produced by an automatic speech

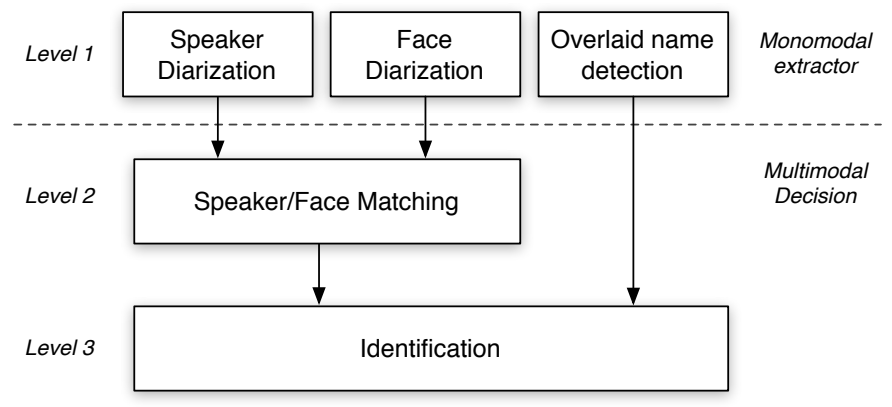

Fig. 2. Overview of the proposed system

recognition (ASR) system, are used: the error rate in speaker identification increases from $16.66 \%$ to $75.15 \%$ [3]. On the other hand, OPNs can be reliably extracted using Optical Character Recognition (OCR) techniques, and their association with people in the videos is easier than analysing whether or not pronounced names in ASR transcripts refer to people appearing in the video.

The identification using OPN, which has started to be investigated 15 years ago [4], has since then raised a large amount of work, especially in face clustering tasks [5], face naming in captioned images [6], and recently, automatic naming within broadcast videos [7], [8], [9], [10], [11]. Regarding broadcast news videos, the use of OPNs raises two main challenges i) the sparsity of OPN information which requires to rely on diarization methods to propagate the identification to all person apparitions ii) the ambiguities that arise when several names and clusters co-occur together.

The work we present there has been carried out during the REPERE French evaluation campaign [12], which focused on multimedia people recognition in television documents. The proposed system, illustrated in Figure 2, consists in a 3-tier architecture.

The first level detects speakers, faces and overlaid person names. The second level associates speakers to faces using cooccurrence statistics between the speaker and the face clusters. In the last level, the overlaid person names are propagated to the speakers, or faces, in order to give, at any time, the identities of the persons in the show. Two identification methods, developped in parallel in different labs, are compared in this paper. The first one relies directly on the co-occurence times between names, faces and speakers and uses a set of rules to assign a name to each monomodal cluster. The second one uses a CRF which jointly performs the naming of 
TABLE I. NUMBER OF KEY FRAMES, SPEAKERS, FACES AND OVERLAID NAMES IN CORPORA

\begin{tabular}{|c|c|ccc|}
\hline Corpus & Key frames & Speakers & Heads & Overlaid names \\
\hline \hline dev & 1229 & 1229 & 1449 & 197 \\
test & 1040 & 1039 & 1201 & 146 \\
\hline both & 2269 & 2268 & 2650 & 343 \\
\hline
\end{tabular}

all person clusters, thus allowing to account for pair-wised constraints and heterogeneous co-occurrence statistics. Two other consortiums participated to this campaign [9], [11]. Our approach differs from those previous works in, first, the choice of using face diarization directly as a pre-process to work both with speaker and face clusters, and second, the CRF-based identification method.

\section{CORPUS}

This study was conducted on two corpora from the REPERE French evaluation campaign [12]. The development corpus (dev) is composed of 28 TV shows. This corpus corresponds to the test corpus of the first evaluation (January 2013). The test corpus (test) is composed of $27 \mathrm{TV}$ shows. It corresponds to the development set of the second evaluation (January 2014). Shows are recorded from the two digital French terrestrial television stations BFM and LCP (respectively MPG2 $720 \times 576$ and MPEG2 $544 \times 576$ ).

Thoses corpora are balanced between prepared speech, with 15 broadcast news, and more spontaneous speech, from 40 political discussions or street interviews. Only a part of the recordings are annotated, giving respectively a total duration of 3 hours for both corpus.

A keyframe of the corpora is annotated every 10 seconds, giving both identity of speakers and faces. The overlaid text corresponding to a target person is also annotated. The Table I reports some statistics about the corpora, and the Fig 1 shows an example of annotation.

\section{LEVEL 1: SPEAKER, FACE AND OPN}

The audio processing, known as the speaker diarization task ( $c f$. sub-section III-A), is performed without any prior information regarding speakers: neither the number of speakers, nor their identities, nor voice samples are available. However, speaker diarization only tags audio segments with anonymous automatically-generated labels. The audio stream is split into small segments, which are clustered speakers. The video processing, called face diarization task ( $c f$. sub-section III-B), consists in the same kind of process as the speaker diarization, except it is performed on face tracks in the video stream instead of speech segments in the audio stream. The OPN set ( $c f$. sub-section III-C) is extracted using, first, an OCR on the video stream, then, a named entity detector which uses external ressources (predefined lists, freebase database, google hits,...).

\section{A. Speaker Diarization}

The speaker diarization system ("who speak when?") is based on the LIUM Speaker Diarization system [13], freely distributed $^{1}$. This system has achieved the best or second best results in the speaker diarization task on French broadcast news

\footnotetext{
${ }^{1}$ http://www-lium.univ-lemans.fr/en/content/liumspkdiarization
}

evaluation campaigns, such as ESTER2, ETAPE (2011) [14] and REPERE (january 2012 and january 2013) [15].

The diarization system is composed of an acoustic Bayesian Information Criterion (BIC)-based segmentation followed by a BIC-based hierarchical clustering. Each cluster represents a speaker and is modeled with a full covariance Gaussian. A Viterbi decoding re-segments the signal using GMMs with 8 diagonal components learned by EM-ML, for each cluster. Segmentation, clustering and decoding are performed with $12 \mathrm{MFCC}+\mathrm{E}$, computed with a $10 \mathrm{~ms}$ frame rate. Music and jingle regions are removed using a Viterbi decoding with 8 GMMs (trained on french broadcast news data) for music, jingle, silence, and speech (with wide/narrow band variants for the last two, and clean/noised/musical background variants for wideband speech).

Gender and bandwidth are detected before transcribing the signal using the LIUM ASR [16]. A new non-speech segmentation is built from the filler words of the transcript. Non-speech segments longer than 0.5 second are removed in the speaker diarization.

In the previous steps, features were used unnormalized in order to preserve information on the background environment, which may help differentiating between speakers. At this point however, each cluster contains the voice of only one speaker, but several clusters can be related to a same speaker. The background environment contribution must be removed from each GMM cluster, through feature gaussianization, in order to obtain a one-to-one relationship between clusters and speakers.

Finally, the system is completed with clustering method based on the i-vectors paradigm and Integer Linear Programming (ILP). This new clustering method is fully described in [17]. The ILP clustering along with i-vectors speaker models gives better results than the usual hierarchical agglomerative clustering based on GMMs and cross-likelihood distances [18].

\section{B. Face Diarization}

The face diarization ("who appears when?") process consists of four main steps. First, shot boundary detection is performed to split the video stream into homogenous video clips. Second, frontal faces are detected within each shot using Viola And Jones algorithm [19]. False alarms are filtered out, based on temporal continuity and skin color detection. Then, face tracking is used to temporally extends the face detections within each shot into face tracks and increase the recall detection rate. Eventually, a face clustering step groups all face tracks that belong to the same person together. This last step is carried out using the method from [20]. It is based on a bottom-up algorithm, which computes the face similarities by combining Speeded Up Robust Features based distances, and statistical models built on block-based DCT features. This method reached state of the art results on the publicly available BUFFY dataset [21].

\section{Overlaid name detection}

The process is based on two steps: the first extracts hypotheses sentences from the video overlaid, the second searches for speaker names in the extracted sentences. The system used [22] to detect texts in the image sequences and 
transcribe it. The error rates of that system on the January 2012 dry-run corpus of the REPERE campaign is about $12 \%$ in terms of character, and $31 \%$ in terms of words.

The name research in overlaids is a task of named entity detection reduced to a single entity type: the "persons". Compared to the same task in journalistic texts or audio transcripts, the announcement of the "persons" in the overlaids is well formatted, making the detection easier. Persons are mostly announced over two written lines, where the first refers to the person identity, and the second, the person function. The identity of a person is generally constituted of a firstname followed by a lastname. In some rare cases, the identity of a person only consists in a firstname, as for people interviewed in the street, or a stage name, as for artists.

Usually, named entity detectors use the linguistic context bordering the named entity to help its detection and categorization. The only context in the video overlaids could be the second line which presents the function. We chose to develop a rule-based identities detection system along with identity dictionaries. In that way, transcripts errors are taken into account much more easily than using a CRF-based method, as it was done in name entity detection. Three identity dictionaries were built:

1) target: the first dictionary includes 7345 identities. Most entries refer to journalists of the processed television stations, political personnalities, athletes and artists recurrently appearing in French media and REPERE training corpus.

2) freebase: The second dictionary is composed of more than 1.7 million of identities extracted from freebase [23]. We only extracted identities of people born after 1900 , as well as those for which the birthdate is unknown.

3) firstname: The third and last dictionary consists in more than 17000 firstnames extracted from a specialized web site.

The detection process is only concerned by the first line of the video overlaids ( $c$ in the following):

1) Rejection step: $c$ is rejected if the number of characters is less than 3 or more than 30 , if $c$ is composed of more than 10 words, or if $c$ includes a keyword belonging to a pre-definite list. That list mostly includes conjugated action verbs. This step aims to reject lines $c$ matching everything else than only an identity, like information messages which start by a person name.

2) $\quad c$ is accepted if it matches an entry from the target dictionary. The search into dictionary is performed using an error tolerance: the identity $n$ of the dictionary is substituted to $c$ if $s(c)>0.8$ (eq. 1) such as:

$$
s(c)=\min _{n \in N}\left(1-\frac{d(c, n)}{\max (l(c), l(n))}\right)
$$

where $N$ is the target dictionary, $d(c, n)$ the Levenshtein distance between $c$ and $n$, and $l(x)$ the string length of $x$.

3) $c$ is accepted if it matches an entry of the freebase dictionary.
Fig. 3. Google roquets for "Laurence Piau"

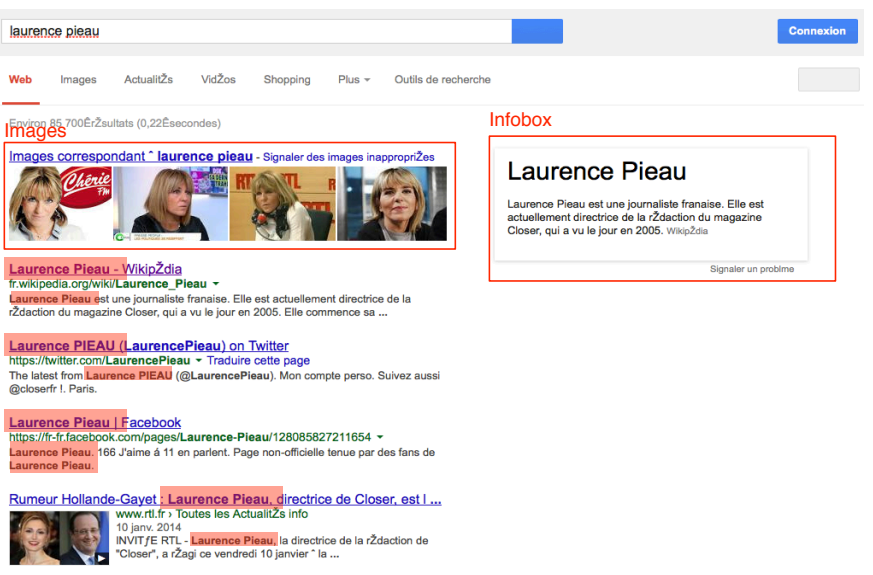

4) Finally, $c$ is accepted if it starts with a firstname available in the firstname dictionary, and if the Google search engine gives clues to confirm the hypothetical identity. The analysis of the HTML page returned by the Google search engine, which answers to a request on $c$, is used to validate, or invalidate the hypothetical identity (Fig. 3). Analysis criteria on the Google HTML page relies on the frequency of $c$ in the page, the presence of pictures associated to $c$, the presence of an informative box describing an individual, and the alternative proposed spelling.

\section{LEVEL 2: MATCHING}

The next step, using the speaker and face diarization, consists in matching the speaker $i \in\{1, \ldots, N\}$ with it's face $j \in\{1, \ldots, M\}$. In the REPERE training corpus, $78.4 \%$ of the speakers have their faces appearing on the video. We made the assumption that the more a speaker voice is heard in the same time a face is seen, the higher the chance that speaker and that face correspond to the same person. Therefore, co-occurrence time between speaker $i$ and face $j$ is computed for each pair $(i, j)$. In the following, we refer to that co-occurrence value as $\delta t(i, j)$. It should be noted that this could be completed with lip activity detection as in [11]. The matching problem can be considered as the search of the largest set of pairs $(i, j)$, for which the sum of the co-occurrences $\delta t(i, j)$ is maximal, and where each speaker $i$ can only be associated to a single face $j$, et vice versa. Formally, this problem can be formulated as an Integer Linear Programming problem, given in 2 , where $x_{i, j}$ is a binary variable equals to 1 when the speaker $i$ is associated with the face $j$ (i.e., $(i, j)$ has been selected).

$$
\begin{array}{ll}
\text { Maximize: } & \sum_{i=1}^{n} \sum_{j=1}^{m} \delta t(i, j) x_{i, j} \\
\text { Subject to: } & \sum_{i=1}^{n} x_{i, j}=1, \quad(i=1, \ldots, n) \\
& \sum_{i=1}^{n} x_{i, j}=1, \quad(j=1, \ldots, m) \\
& x_{i, j} \in 0,1
\end{array}
$$


The number of solution of this problem is $p$ !, with $p$ the higher number between speaker and face cluster numbers. However, an optimal solution to that matching problem can be found in a polynomial time using the Kuhn-Munkres algorithm [24]. In order to use the Kuhn-Munkres algorithm, we transform the maximisation problem into a minimisation problem, by changing the co-occurrence value in:

$$
\delta t^{\prime}(i, j)=\max _{i, j} \delta t(i, j)-\delta t(i, j)
$$

After applying the Kuhn-Munkres algorithm, the speaker clusters which have been matched to face clusters are renamed with the same label, in their respective diarization segmentations. To account for segment boundary errors, speakers and faces are only matched if they overlap more than 0.1 second.

\section{LEVEL 3: IDENTIFICATION}

At this level, audio and video diarization have been already performed. Several speaker clusters have been associated to faces clusters. In addition, the OPNs have been extracted. OPNs are characterized by their appearance and disappearance times, which make them constitute segments. We propose two identification methods. The first one, described in subsection V-A, uses co-occurrence statistics between clusters and OPNs to choose the best identity for each clusters, without any consideration about the identities attributed to the other clusters. The second method ( $c f$. sub-section V-B), inspired from [25], relies on Conditional Random Fields (CRFs) and performs a joint identification of all person clusters (speakers and faces), allowing to account for uniqueness constraints and co-occurrence statistics between clusters and OPNs (see [26] for more details).

\section{A. Direct identification}

This identification method is first applied to speaker clusters. For speaker clusters that have been associated to a face cluster, the identity is propagated to both speaker and face clusters. Then, this identification method is applied to the face clusters for which no speaker cluster have been matched.

As for the speaker/face clusters matching step ( $c f$. section IV), we used the co-occurrence times $\delta t\left(C_{i}, P_{j}^{k}\right)$ between the occurrences $P_{j}^{k}$ with $k=1 \ldots K_{j}$ of a OPN $P_{j}$ from the set of OPN $P=\left\{P_{j}, j=1 \ldots N^{P}\right\}$, and the segments of a speaker or face cluster $C_{i}$. Each $\delta t\left(C_{i}, P_{j}^{k}\right)$ is normalized by appearance duration $d_{k}^{j}$ of the occurrence $P_{j}^{k}$. Each occurrence score of the name $P_{j}$ are then summed. The cluster $C_{i}$ is therefore identified by the name $P_{j}$ giving the highest score among the OPN set $P$, which refers to all the OPN in cooccurrence with the cluster $C_{i}$ (equation 4 ).

$$
C_{i}=\underset{P_{j} \in P}{\operatorname{argmax}} \sum_{k=1}^{K_{j}} \frac{\delta t\left(C_{i}, P_{j}^{k}\right)}{d_{k}^{j}}
$$

$d_{k}^{j}$, which is the appearance duration normalization of the occurrence $P_{j}^{k}$, favours a name $P_{j}$ which recurrently appear during the various person $C_{i}$ interventions, instead of favouring the total appearance durations of OPNs related to the person $C_{i}$.
Fig. 4. Speaker diarization, Face diarization et OPN

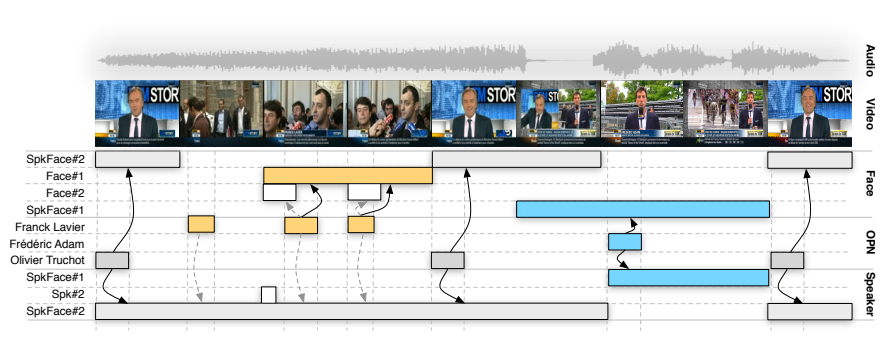

\section{B. CRF identification}

CRF models enable to efficiently combines heterogeneous statistics by and have been used in a speaker identification task using transcripts [27]. Another advantage is the possibility to introduce pairwise relationships between cluster pairs. Let us denote by $C^{a v}=\left\{C_{i}^{a v}, i=1 \ldots N^{C^{a v}}\right\}$ the set of audiovisual clusters obtained after the speaker/face matching step described in Section IV. Here, each cluster $C_{i}^{a v}$ consists either in a speaker/face pair, or a face alone, or a speaker alone. Conversely to the previous method which takes a decision about each mono-modal cluster separately, that method considers audiovisual cluster pairs, thus allowing for more meaningful co-occurrence statistics.

The identification can be seen as an estimation of the label field $E=\left\{e_{i}, i=1 \ldots N^{C^{a v}}\right\}$, such as the label $e_{i}$ corresponds to the name of the cluster $C_{i}^{a v}$. $e_{i}$ takes value from the set of OPNs $P$, augmented by an anonymous label which should be assigned to anonymous persons. Let $\mathrm{G}$ be an undirected graph over the set of random variables $C^{a v}, P$, and $E$. We then seek to maximize the CRF posterior probability $P\left(E \mid C^{a v}, P\right)$ defined as:

$$
P\left(E \mid C^{a v}, P\right)=\frac{1}{Z\left(P, C^{a v}\right)} \times \exp \left\{\sum_{i=1}^{6} \sum_{C l q \in G_{i}} \lambda_{i} f_{i}(C l q)\right\}
$$

where $Z\left(P, C^{a v}\right)$ is a normalization constant, and each triplet $\left(f_{i}, G_{i}, \lambda_{i}\right)$ is composed of a feature function $f_{i}$, the set $G_{i}$ of cliques where this function is defined, and its CRF weight $\lambda_{i}$ learned at training time.

This naming model exploits four different co-occurrence statistics between clusters and OPNs. First, for each triplet $\left(e_{i}, C_{i}^{a v}, P_{j}\right)$, we define:

$$
f_{\text {audio }}\left(e_{i}, C_{i}^{a v}, P_{j}\right)=\sum_{k=1}^{K_{j}} \frac{\delta t\left(C_{i}^{a}, P_{j}^{k}\right)}{d_{k}^{j}}
$$

Where $C_{i}^{a}$ is the audio part of $C_{i}^{a v}$ (which can be null). We define similarly two other feature functions for the visual part of $C_{i}^{a v}: f_{v_{-}} a l o n e$ which counts the co-occurrences when the face is alone in the image, and $f_{v_{-} m u l t i}$ which accounts for multi-face images. Thus, the CRF will learn a different $\lambda$ weight for each case and will be able to weight each type of information. Eventually, we followed the assumption that a person does not appear or speak before the first apparition of his name in an OPN, and defined a function $f_{\text {before }}\left(e_{i}, C_{i}^{a v}, P\right)$ 
which returns the number of audio segments from cluster $C_{i}^{a v}$ which occurs before the first apparition of the name $e_{i}$. We also introduce prior knowledge over the anonymous label by defining a fifth feature function $f_{a n o}\left(e_{i}, C_{i}^{a v}\right)$ which returns 1 if $e_{i}$ is the anonymous label. Lastly, we define a uniqueness function $f_{u n i q}\left(e_{i}, C_{i}^{a v}, e_{j}, C_{j}^{a v}\right)$ over visually overlapping clusters. For such pair $C_{i}^{a v}, C_{j}^{a v}$ :

$$
f_{\text {uniq }}\left(C_{i}^{a v}, C_{j}^{a v}, e_{i}, e_{j}\right)= \begin{cases}- \text { Inf } & \text { if } e_{i}=e_{j} \\ 0 & \text { otherwise }\end{cases}
$$

\section{EXPERIMENTS}

\section{A. Evaluation metrics}

The evaluation metric chosen to measure the identification performance is the official REPERE Estimated Global Error Rate (EGER). This metric is defined as follow:

$$
E G E R=\frac{\# f a+\# \text { miss }+\# \text { conf }}{\# \text { total }}
$$

where \#total is the number of persons to be detected, \#conf the number of persons wrongly identified, \#miss the number of missed persons and $\# f a$ the number of false alarms. It should be noted that this metric does not take into account the spatial position of the faces but rather focus on evaluating the ability of the system to answer the question "Who is seen?" and "Who is speaking".

To evaluate the impact of the identification, we report the Diarization Error Rate (DER). The metric was introduced by the NIST as the fraction of speaking time which is not attributed to the correct speaker using the best match between references and hypothesis speaker labels. Both DER and EGER are computed using the scoring tool developed by the $\mathrm{LNE}^{2}$ as part of the REPERE campaigns.

\section{B. Overlaid person name detection results}

The Table II shows the evaluation results of the overlaid person names, for the both corpora. The EGER is $15.5 \%$. Most of the errors are due to missed overlaid names, which were too close to the overlaid segment boundaries.

The 8 confusion errors come from erroneous transcripts, thus generating a substitution of the transcript name with the most similar target name.

TABLE II. EVALUATION OF OVERLAID NAME DETECTION

\begin{tabular}{|c|c|ccc|}
\hline Corpus & EGER & \#conf & \#miss & \#fa \\
\hline \hline DEV & $14.2 \%$ & 0 & 22 & 6 \\
TEST & $17.1 \%$ & 8 & 16 & 1 \\
\hline both & $15.5 \%$ & 8 & 38 & 7 \\
\hline
\end{tabular}

\section{Association and Identification results}

The speaker DER is $13.12 \%$ on the dev set and $15.00 \%$ on the test set. A third of DER errors come from missed detection. The reason is that BFM is a television station which continuously broadcast, frequently using jingles to capture the public attention. Those are detected as non-speech areas, even if the host is announcing headlines. Regarding the Face

\footnotetext{
${ }^{2}$ The French National Laboratory of Metrology and Testing
}

diarization, the DER is $77 \%$ person on the dev set and $77 \%$ on the test set. Overall $75 \%$ of the errors come from miss detections due to non profile faces and person seen from the back. Qualitatively, we found that the face diarization method provides pure face clusters, but might split one person into different clusters if the head pose variations are important.

TABLE III. PRECISION, RECALL AND F-MESURE ON DEV AND TEST CORPORA. RESULTS ARE REPORTED WHILE EVALUATING THE SPEAKER

\begin{tabular}{|c|c|c|c|c|}
\hline Corpus & EGER & \#conf & \#miss & $\#$ fa \\
\hline \multicolumn{5}{|c|}{ (1) DEV: Oracle } \\
\hline Speaker & $20.2 \%$ & $25(2.0 \%)$ & $206(16.8 \%)$ & $17(1.4 \%)$ \\
\hline Head & $31.5 \%$ & $8(0.5 \%)$ & $446(30.8 \%)$ & $3(0.2 \%)$ \\
\hline Spk + Head & $26.3 \%$ & $33(1.2 \%)$ & $652(24.3 \%)$ & $20(0.7 \%)$ \\
\hline \multicolumn{5}{|c|}{ (2) TEST: Oracle } \\
\hline Speaker & $29.9 \%$ & $23(2.2 \%)$ & $284(27.3 \%)$ & $4(0.4 \%)$ \\
\hline Head & $38.6 \%$ & $2(0.2 \%)$ & $461(38.4 \%)$ & $0(0 \%)$ \\
\hline Spk + Head & $34.6 \%$ & $25(1.1 \%)$ & $745(33.3 \%)$ & $4(0.2 \%)$ \\
\hline \multicolumn{5}{|c|}{ (3) DEV: System "Direct" with OPN groundtruth } \\
\hline Speaker & $25.0 \%$ & $114(11.7 \%)$ & $181(18.4 \%)$ & $12(0.8 \%)$ \\
\hline Head & $37.5 \%$ & $49(3.4 \%)$ & $465(32.1 \%)$ & $29(2 \%)$ \\
\hline Spk + Head & $31.7 \%$ & $163(6.1 \%)$ & $646(24.1 \%)$ & $41(1.5 \%)$ \\
\hline \multicolumn{5}{|c|}{ (4) TEST System "Direct" with OPN groundtruth } \\
\hline Speaker & $37.8 \%$ & $152(14.6 \%)$ & $236(22.7 \%)$ & $5(0.5 \%)$ \\
\hline Head & $45.0 \%$ & $51(4.3 \%)$ & $482(40.1 \%)$ & $7(0.6 \%)$ \\
\hline Spk + Head & $41.7 \%$ & $203(9.1 \%)$ & $718(32.1 \%)$ & $12(0.5 \%)$ \\
\hline \multicolumn{5}{|c|}{ (5) DEV: System "Direct" } \\
\hline Speaker & $25.0 \%$ & $114(11.7 \%)$ & $181(14.7 \%)$ & $12(1.0 \%)$ \\
\hline Head & $37.4 \%$ & $50(3.5 \%)$ & $465(32.1 \%)$ & $27(1.8 \%)$ \\
\hline Spk + Head & $31.7 \%$ & $164(6.1 \%)$ & $646(24.1 \%)$ & $39(1.5 \%)$ \\
\hline \multicolumn{5}{|c|}{ (6) TEST: System "Direct" } \\
\hline Speaker & $39.2 \%$ & $166(16 \%)$ & $236(22.7 \%)$ & $5(0.5 \%)$ \\
\hline Head & $46.4 \%$ & $68(5.7 \%)$ & $482(40.1 \%)$ & $7(0.6 \%)$ \\
\hline Spk + Head & $43.0 \%$ & $234(10.5 \%)$ & $718(32.0 \%)$ & $12(0.5 \%)$ \\
\hline \multicolumn{5}{|c|}{ (7) DEV: System "CRF" } \\
\hline Speaker & $24.9 \%$ & $102(8.3 \%)$ & $192(15.6 \%)$ & $12(1.0 \%)$ \\
\hline Head & $38.4 \%$ & $55(3.4 \%)$ & $480(33.1 \%)$ & $21(1.4 \%)$ \\
\hline Spk + Head & $32.2 \%$ & $157(5.9 \%)$ & $672(25.1 \%)$ & $33(1.2 \%)$ \\
\hline \multicolumn{5}{|c|}{ (8) TEST: System "CRF" } \\
\hline Speaker & $37.2 \%$ & $150(14.4 \%)$ & $233(22.4 \%)$ & $4(0.4 \%)$ \\
\hline Head & $46.0 \%$ & $54(4.5 \%)$ & $492(41.0 \%)$ & $7(0.6 \%)$ \\
\hline Spk + Head & $42.0 \%$ & $204(9.1 \%)$ & $725(32.4 \%)$ & $11(0.5 \%)$ \\
\hline
\end{tabular}
ONLY, THE HEAD ONLY AND BOTH

The identification results for the different systems are presented in Table III. Table (1-2) is an oracle simulating the best possible results we would reach with perfect diarization and identification systems, while keeping automatic speech segmentation, face and OPN detections. For the speaker part, errors are mostly due to journalist off-voices, which are not announced by OPNs. Considering the faces, miss errors are mainly divided into non-frontal faces and figurative persons not announced by OPNs. The few confusions and false alarms are due to inaccuracies of segment boundaries. In addition to provide an upper bound for the identification results, those tables highlight the differences among the dev and test sets. The identification on the test set is considerably more difficult with an increase of the EGER from $26.3 \%$ to $34.6 \%$.

The tables (3-4) and (5-6) present the identification results of the direct method. The system in tables (3-4) uses OPN ground truth while the other system uses the OPNs automatically extracted. While performances are stable for the dev test, the use of automatic OPN detection increase the EGER from $41.7 \%$ to $43 \%$. This change could probably be explained by the confusions errors in the OPN detections only observed in the test set, in table II.

The increase in the miss rate, with respect to the oracle, is mainly due to diarization errors where a person is split into several clusters. Indeed, those smaller clusters are likely to not 
co-occur with any OPN, and thus, it is not possible to identify them. Confusion errors arise when a cluster co-occurs with wrong names.

Regarding comparisons with the Direct method and the CRF method (tables 5 to 8), two differences can be highlighted. First, the CRF tends to give a better performance on the speaker identification, with an absolute reduction of the EGER from $39.2 \%$ to $37.2 \%$ on the test set. Improvements mainly come from a reduction of the confusion errors, meaning that the considered co-occurrence statistics, and the joint audiovisual decision process used by the CRF, can help to solve ambiguities. Secondly, considering the head identification, the Direct method gives more confusion and less miss errors. Actually, this method try to identify all possible faces, and may label two co-occurring clusters with the same name. We found this strategy optimal for the EGER metric which does not take into account the spatial positions of the faces as explained previously. The CRF, by using the uniqueness constraint, provides a more accurate naming at the cost of more miss errors. Although performances are similar regarding EGER metric, the CRF results are more suitable for other tasks such as collecting faces of a given person for unsupervised model training.

\section{CONCLUSIONS}

In this work, a method for identifying people using overlaid person names in TV shows has been described. Results shows that such OPNs can be reliably extracted and used to identify face and speaker clusters. Two approaches are explored to associate names and clusters. The first identification method relies on co-occurrence times between overlay person names and speaker/face clusters; the second method uses a CRF to jointly identify the speaker and the face clusters. By jointly exploiting the audiovisual information and additional context clues, the CRF provides the best results, mainly by solving ambiguous speaker cases where a same person co-occurs with different OPNs.

The difference of performances between the oracle and the automatic systems show that there is room for improvements in the diarization and identification steps. First, the speaker and face diarizations could be optimised for the identification tasks, for instance by taking into account the OPNs information [28]. Second, the CRF method could easily be extented to include biometrics models learned in an supervised or unsupervised way [29], [30]. Last but not least, saliency and namedness features could be used in multi-face images to improve name/face association.

\section{ACKNOWLEDGMENT}

This research was supported by ANR (French National Research Agency) under contract number ANR-2010-CORD101-01 (SODA project), as well as by the European Union (project FP7-611057 EUMSSI).

\section{REFERENCES}

[1] L. Canseco-Rodriguez, L. Lamel, and J.-L. Gauvain, "A comparative study using manual and automatic transcriptions for diarization," in Proc. of ASRU, 2005.

[2] S. Tranter, "Who really spoke when? Finding speaker turns and identities in broadcast news audio," in Proc of ICASSP, 2006.
[3] V. Jousse, S. Petit-Renaud, S. Meignier, Y. Estève, and C. Jacquin, "Automatic named identification of speakers using diarization and ASR systems," in Proc. of ICASSP, 2009.

[4] S. Satoh, Y. Nakamura, and T. Kanade, "Name-it: Naming and detecting faces in news videos," Proc. of IEEE Multimedia, 1999.

[5] M. Everingham, J. Sivic, and A. Zisserman, "Hello! my name is... buffy automatic naming of characters in tv video," in Proc. of BMVC, 2006.

[6] T. Berg, A. Berg, J. Edwards, M. Maire, R. White, Y. Teh, E. LearnedMiller, and . Forsyth, "Names and faces in the news," in Proc. of CVPR, 2004.

[7] M. Pham, P. and T. Tuytelaars, "Naming persons in news video with label propagation," in Proc. of ICME, 2010.

[8] B. Jou, H. Li, J. G. Ellis, D. Morozoff-Abegauz, and S.-F. Chang, "Structured exploration of who, what, when, and where in heterogeneous multimedia news sources," in Proc.of ACM Multimedia, 2013.

[9] J. Poignant, L. Besacier, V. B. Le, S. Rosset, and G. Quénot, "Unsupervised naming of speakers in broadcast tv: using written names, pronounced names or both?" 2013.

[10] J. Poignant, H. Bredin, V. Le, L. Besacier, C. Barras, G. Quénot et al., "Unsupervised speaker identification using overlaid texts in tv broadcast," in Proc. of Interspeech, 2012.

[11] M. Bendris, B. Favre, D. Charlet, G. Damnati, G. Senay, R. Auguste, and J. Martinet, "Unsupervised face identification in tv content using audio-visual sources," in Proc. of CBMI, 2013.

[12] J. Kahn, O. Galibert, L. Quintard, M. Carré, A. Giraudel, and P. Joly, "A presentation of the repere challenge," in Proc. of CBMI, 2012.

[13] M. Rouvier, G. Dupuy, P. Gay, E. Khoury, T. Merlin, and S. Meignier, "An open-source state-of-the-art toolbox for broadcast news diarization," in Proc. of Interspeech, 2013.

[14] G. Gravier, G. Adda, N. Paulson, M. Carré, A. Giraudel, O. Galibert et al., "The etape corpus for the evaluation of speech-based tv content processing in the french language," in Proc. of ICLREC, 2012.

[15] O. Galibert and J. Kahn, "The first official REPERE evaluation," in Proc. of SLAM, Marseille, France, 2013.

[16] P. Deléglise, Y. Estève, S. Meignier, and T. Merlin, "Improvements to the lium french asr system based on cmu sphinx: what helps to significantly reduce the word error rate?" in Proc. of Interspeech, 2009.

[17] M. Rouvier and S. Meignier, "A global optimization framework for speaker diarization," in Proc. of Odyssey, 2012.

[18] C. Barras, X. Zhu, S. Meignier, and J.-L. Gauvain, "Improving speaker diarization," in Proc. of DARPA RT04, 2004.

[19] P. Viola and M. Jones, "Robust real-time face detection," Proc. of IJCV, 2004.

[20] E. Khoury, P. Gay, and J. Odobez, "Fusing matching and biometric similarity measures for face diarization in video," in Proc. of ICMR, 2013.

[21] M. Guillaumin, J. Verbeek, and C. Schmid, "Is that you? metric learning approaches for face identification," in Proc of ICCV, 2009.

[22] D. Chen and J. Odobez, "Video text recognition using sequential monte carlo and error voting methods," Pattern Recogn. Lett., vol. 26, no. 9, pp. 1386-1403, 2005.

[23] K. Bollacker, C. Evans, P. Paritosh, T. Sturge, and J. Taylor, "Freebase: A collaboratively created graph database for structuring human knowledge," in Proc. of ACM SIGMOD, 2008.

[24] J. Munkres, "Algorithms for the assignment and transportation problems," Proc. of JSIAM, 1957.

[25] P. Gay, E. Khoury, S. Meignier, J.-M. Odobez, and P. Deleglise, "A conditional random field approach for audio-visual people diarization," in IEEE International Conference on Acoustics, Speech and Signal Processing (ICASSP 2014), 2014.

[26] _ "Face identification from overlaid texts using local face recurrent patterns and crf models," in IEEE International Conference on Image Processing (ICIP), 2014.

[27] C. Ma, P. Nguyen, and M. Mahajan, "Finding speaker identities with a conditional maximum entropy model," in Proc. of ICASSP, 2007.

[28] H. Bredin and J. Poignant, "Integer linear programming for speaker diarization and cross-modal identification in tv broadcast," 2013. 
[29] C. Lailler, G. Dupuy, M. Rouvier, and S. Meignier, "Semi-supervised and unsupervised data extraction targeting speakers: From speaker roles to fame?" in In Proc. of SLAM, 2013.

[30] C. Liu, S. Jiang, and Q. Huang, "Naming faces in broadcast news video by image google," in Proc. of ACM Multimedia. 